\title{
A New Approach for Guaranteed Ellipsoidal State Estimation
}

\author{
S. Ben Chabane* C. Stoica Maniu* T. Alamo** \\ E.F. Camacho ${ }^{* *}$ D. Dumur * \\ * SUPELEC Systems Sciences (E3S) - Automatic Control \\ Department, 3 rue Joliot Curie, F-91190, Gif-sur-Yvette cedex, France \\ (e-mail: \{sofiane.benchabane,cristina.stoica,didier.dumur\}@supelec.fr). \\ ** Department of Ingeniería de Sistemas y Automática, Universidad de \\ Sevilla, Camino de los Descubrimientos, 41092 Sevilla, Spain \\ (e-mail: alamo@cartuja.us.es, eduardo@esi.us.es).
}

\begin{abstract}
This paper proposes a new ellipsoid-based guaranteed state estimation approach for linear discrete-time systems with bounded perturbations and bounded measurement noise. This approach is based on the minimization of the radius of the ellipsoidal state estimation set. Firstly, the ellipsoidal state estimation is computed by off-line solving a Linear Matrix Inequality optimization problem. Secondly, a new online method is developed in order to improve the accuracy of the estimation but it leads to an increase of the online computation load. A new scaling technique is proposed to reduce the computation time, while keeping a good accuracy of the state estimation. An illustrative example is analyzed in order to show the advantages of the proposed approach.
\end{abstract}

Keywords: Set-membership estimation, ellipsoidal set, linear systems, Linear Matrix Inequality.

\section{INTRODUCTION}

Knowing the system state is crucial for solving many control problems. However, in many cases, the full state is not directly measurable and an estimation of the system state is required. Stochastic and set membership methods for state estimation have been proposed in the literature.

The stochastic approaches (e.g. Kalman filter theory see Kalman (1960)) assume that the perturbations and measurement noises have a known (usually Gaussian) distribution. This assumption is in many cases difficult to validate. Thus, it may be more realistic to assume that the perturbations and measurement noises are unknown but bounded. This leads to the problem of guaranteed state estimation introduced by Schweppe (1968), Wistenhausen (1968). Set-membership approaches have been developed in Bertsekas and Rhodes (1971), Fogel and Huang (1982). In these approaches, the idea is to compute a compact set that guarantees to contain the set of states that are consistent with the model of the system, the realization of the measurement and the bounded perturbations and measurement noises. To implement set-membership estimation techniques, several sets are used: ellipsoids (Kurzhanski and Vályi (1996), Durieu et al. (2001), Polyak et al. (2004), Daryin et al. (2006), Daryin and Kurzhanski (2012), Chernousko (1994)), polytopes (Walter and PietLahanier (1989)), parallelotopes (Chisci et al. (1996)), and zonotopes (Puig et al. (2001), Combastel (2003), Alamo et al. (2005), Le et al. (2013)).

Ellipsoids are widely used due to the simplicity of their formulation and resulting estimation stability properties (Hu and Lin (2003)). To minimize the size of the esti- mation ellipsoidal set, two methods are mainly considered (see Durieu et al. (2001)). Firstly, the determinant-based criterion is minimized, which is equivalent to minimize the volume of the ellipsoidal set. Secondly, the minimization of the trace criterion, which is equivalent to minimize the sum of squares of the half length of the axes of the ellipsoid, is considered in the literature. These two methods offer low complexity, but with a loss of accuracy compared to the polytopic estimation (Durieu et al. (2001)).

In this paper, a new approach for guaranteed state estimation for Multi-Input Multi-Output linear systems by minimizing the radius of the ellipsoidal estimation set is proposed. First, off-line solving a Linear Matrix Inequality (LMI) problem leads to a constant observer gain matrix related to the center of the ellipsoid. Second, to improve the state estimation, this observer gain is updated online at each iteration by solving an LMI optimization problem. This leads to a more accurate estimation than the off-line method, but with a significant increase of the computation burden. Third, in order to reduce the computation load, while keeping a suitable level of the estimation accuracy, a new scaling technique is further proposed.

The paper is organized as follows. Section 2 presents a new off-line method for guaranteed state estimation using ellipsoids. Based on a first solution obtained off-line, Sections 3 proposes an online approach that will improve the convergence of the ellipsoidal state estimation method. A scaling technique is proposed in Section 4 in order to reduce the computation time of the online method. An illustrative example comparing the proposed methods is analyzed in Section 5. Finally, some concluding remarks and perspectives are drawn. 


\section{Notations and definitions}

An interval $[a, b]$ is defined as the set $\{x \in \mathbb{R}: a \leq x \leq b\}$. A unitary interval is $\mathbf{B}=[-1,1]$. A box $\left(\left[a_{1}, b_{1}\right], \ldots,\left[a_{n}, b_{n}\right]\right)^{\top}$ is an interval vector. A unitary box in $\mathbb{R}^{m}$, denoted by $\mathbf{B}^{m}$, is a box composed by $m$ unitary intervals.

A symmetric matrix $M=M^{\top} \in \mathbb{R}^{n \times n}$ is called a positive definite matrix (resp. negative definite matrix), denoted $M \succ 0$ (resp. $M \prec 0$ ), if $z^{\top} M z>0$ (resp. $z^{\top} M z<0$ ) for all non-zero vectors $z$ with real entries $\left(z \in \mathbb{R}^{n} \backslash\left\{0_{n}\right\}\right)$.

Given a matrix $P=P^{\top} \succ 0$, a vector $\bar{x} \in \mathbb{R}^{n_{x}}$ and a scalar $\rho \in \mathbb{R}_{+}^{*}$, the bounded ellipsoid $\mathcal{E}(P, \bar{x}, \rho)$ is the set:

$$
\mathcal{E}(P, \bar{x}, \rho)=\left\{x \in \mathbb{R}^{n_{x}}:(x-\bar{x})^{\top} P(x-\bar{x}) \leq \rho\right\},
$$

where $P$ is the shape matrix of the ellipsoid, $\bar{x}$ its center and $\rho$ its radius.

A polyhedral set in a finite-dimensional euclidean space is the intersection of a finite number of closed half-spaces. Given a bounded polyhedral set $\mathcal{X}$, denote by $\mathcal{V}_{\mathcal{X}}$ the set of its vertices.

\section{GUARANTEED ELLIPSOIDAL STATE ESTIMATION}

Consider the following discrete-time Linear Time Invariant (LTI) system:

$$
\left\{\begin{array}{l}
x_{k+1}=A x_{k}+B u_{k}+E \omega_{k} \\
y_{k}=C x_{k}+D u_{k}+F \omega_{k}
\end{array}\right.
$$

where $x_{k} \in \mathbb{R}^{n_{x}}$ is the state vector of the system, $u_{k} \in \mathbb{R}^{n_{u}}$ is the input vector, and $y_{k} \in \mathbb{R}^{n_{y}}$ is the measured output vector at sample time $k$. The vector $\omega_{k} \in \mathbb{R}^{n_{x}+n_{y}}$ contains the state perturbations and the measurement perturbations (noise, offset, etc.), which can be non-correlated. Matrices $A, B, C, D, E$, and $F$ have the appropriate dimensions, with the pair $(C, A)$ detectable and the pair $(A, B)$ stabilizable. It is assumed that the perturbations $\omega_{k}$ are bounded by the unitary interval $\mathbf{B}^{n_{x}+n_{y}}$ and the initial state $x_{0}$ is bounded by the ellipsoid:

$\mathcal{E}\left(P_{0}, \bar{x}_{0}, \rho_{0}\right)=\left\{x \in \mathbb{R}^{n_{x}}:\left(x-\bar{x}_{0}\right)^{\top} P_{0}\left(x-\bar{x}_{0}\right) \leq \rho_{0}\right\}$ with $\bar{x}_{0}$ the initial nominal state. In fact, the matrices $E$ and $F$ represent weights for the normalized perturbations $\omega_{k} \in \mathbf{B}^{n_{x}+n_{y}}$.

Given an ellipsoidal estimation set for $x_{k}$ of the form $\mathcal{E}\left(P, \bar{x}_{k}, \rho_{k}\right)$, with $\bar{x}_{k}$ the nominal estimated state, the aim of this paper is to provide an ellipsoidal state estimation set for $x_{k+1}$ of the form $\mathcal{E}\left(P, \bar{x}_{k+1}, \rho_{k+1}\right)$ in such a way that:

$$
\rho_{k+1} \leq \beta \rho_{k}+\sigma
$$

where $\beta \in(0,1)$ and $\sigma$ is a bounded positive scalar $(\sigma>0)$. Imposing this condition allows us to guarantee the nonincreasing property of the ellipsoidal radius. A similar condition is considered by Le et al. (2013) for zonotopic guaranteed state estimation.

Finding a guaranteed ellipsoid which contains the state vector $x_{k}$ at each sampling time $k$ is formulated by the following theorem.

Theorem 1. Consider an initial state vector $x_{0}$ and assume that $x_{k} \in \mathcal{E}\left(P, \bar{x}_{k}, \rho_{k}\right)$ at time $k$. Given a scalar $\beta \in$ $(0,1)$, if there exist a symmetric positive definite matrix $P=P^{\top} \succ 0$ in $\mathbb{R}^{n_{x} \times n_{x}}$, a matrix $Y \in \mathbb{R}^{n_{x} \times n_{y}}$ and a scalar $\sigma>0$ for which the following LMI holds for every $\omega_{k} \in \mathcal{V}_{\mathbf{B}^{n_{x}+n_{y}}}$ :

$$
\left[\begin{array}{ccc}
\beta P & 0 & A^{\top} P-C^{\top} Y^{\top} \\
0 & \sigma & \omega_{k}^{\top}\left(E^{\top} P-F^{\top} Y^{\top}\right) \\
P A-Y C & (P E-Y F) \omega_{k} & P
\end{array}\right] \succ 0
$$

then the system state $x_{k+1}$ at time $k+1$ is guaranteed to belong to the ellipsoid $\mathcal{E}\left(P, \bar{x}_{k+1}, \rho_{k+1}\right), \forall \omega_{k} \in \mathbf{B}^{n_{x}+n_{y}}$, with the following notations:

$$
\begin{aligned}
Y & =P L, \\
\bar{x}_{k+1} & =A \bar{x}_{k}+B u_{k}+L\left(y_{k}-C \bar{x}_{k}-D u_{k}\right), \\
\rho_{k+1} & \leq \beta \rho_{k}+\sigma .
\end{aligned}
$$

Proof: Denote by $z_{k}=x_{k}-\bar{x}_{k}$ the error between the real state and the nominal estimated state at time $k$. Computing the error $z_{k+1}$ at the next time instant leads to:

$$
z_{k+1}=(A-L C) z_{k}+(E-L F) \omega_{k}=A_{L} z_{k}+\eta_{k},
$$

with $A_{L}=A-L C$ and $\eta_{k}=(E-L F) \omega_{k}$.

To prove the result of Theorem 1, we will show that:

$$
z_{k}^{\top} P z_{k} \leq \rho_{k} \Rightarrow z_{k+1}^{\top} P z_{k+1} \leq \rho_{k+1} \leq \beta \rho_{k}+\sigma .
$$

Denote $F_{0}\left(z_{k}\right)=\rho_{k+1}-\left(A_{L} z_{k}+\eta_{k}\right)^{\top} P\left(A_{L} z_{k}+\eta_{k}\right)=$ $\rho_{k+1}-z_{k+1}^{\top} P z_{k+1}$ and $F_{1}\left(z_{k}\right)=\rho_{k}-z_{k}^{\top} P z_{k}$. Using the S-Procedure (Boyd et al. (1994)) detailed in Appendix, with $p=1$, expression (9) is verified if there exists $\mu>0$ such that $F_{0}\left(z_{k}\right)-\mu F_{1}\left(z_{k}\right) \geq 0, \forall \omega_{k} \in \mathbf{B}^{n_{x}+n_{y}}$, which is equivalent to:

$z_{k+1}^{\top} P z_{k+1}+\mu\left(\rho_{k}-z_{k}^{\top} P z_{k}\right) \leq \rho_{k+1}, \quad \forall \omega_{k} \in \mathbf{B}^{n_{x}+n_{y}}$. From $\rho_{k+1} \leq \beta \rho_{k}+\sigma$, it infers that:

$z_{k+1}^{\top} P z_{k+1}+\mu\left(\rho_{k}-z_{k}^{\top} P z_{k}\right) \leq \beta \rho_{k}+\sigma, \quad \forall \omega_{k} \in \mathbf{B}^{n_{x}+n_{y}}$.

Fixing the value of $\mu$ equal to $\beta$, we obtain:

$z_{k+1}^{\top} P z_{k+1}+\beta\left(\rho_{k}-z_{k}^{\top} P z_{k}\right) \leq \beta \rho_{k}+\sigma, \quad \forall \omega_{k} \in \mathbf{B}^{n_{x}+n_{y}}$. Replacing the term $z_{k+1}$ with the equation (8), the following inequality is obtained:

$\left(A_{L} z_{k}+\eta_{k}\right)^{\top} P\left(A_{L} z_{k}+\eta_{k}\right)-\beta z_{k}^{\top} P z_{k} \leq \sigma, \quad \forall \omega_{k} \in \mathbf{B}^{n_{x}+n_{y}}$. This is equivalent to:

$z_{k}^{\top} A_{L}^{\top} P A_{L} z_{k}+\eta_{k}^{\top} P \eta_{k}+2 \eta_{k}^{\top} P A_{L} z_{k}-\beta z_{k}^{\top} P z_{k}-\sigma \leq 0$, $\forall \omega_{k} \in \mathbf{B}^{n_{x}+n_{y}}$, which can be rewritten as:

$\left[\begin{array}{c}z_{k} \\ 1\end{array}\right]^{\top}\left[\begin{array}{cc}A_{L}^{\top} P A_{L}-\beta P & A_{L}^{\top} P \eta_{k} \\ \eta_{k}^{\top} P A_{L} & -\sigma+\eta_{k}^{\top} P \eta_{k}\end{array}\right]\left[\begin{array}{c}z_{k} \\ 1\end{array}\right] \leq 0$,

$\forall \omega_{k} \in \mathbf{B}^{n_{x}+n_{y}}, \forall z_{k} \in \mathbb{R}^{n_{x}}$. This is satisfied if:

$\left[\begin{array}{cc}-A_{L}^{\top} P A_{L}+\beta P & -A_{L}^{\top} P \eta_{k} \\ -\eta_{k}^{\top} P A_{L} & \sigma-\eta_{k}^{\top} P \eta_{k}\end{array}\right] \succ 0, \quad \forall \omega_{k} \in \mathbf{B}^{n_{x}+n_{y}}$, which is equivalent to

$\left[\begin{array}{cc}\beta P & 0 \\ 0 & \sigma\end{array}\right]-\left[\begin{array}{c}A_{L}^{\top} P \\ \eta_{k}^{\top} P\end{array}\right] P^{-1}\left[\begin{array}{ll}P A_{L} & P \eta_{k}\end{array}\right] \succ 0, \quad \forall \omega_{k} \in \mathbf{B}^{n_{x}+n_{y}}$. Applying Schur complement (Boyd et al. (1994)) leads to: $\left[\begin{array}{ccc}\beta P & 0 & A_{L}^{\top} P \\ 0 & \sigma & \eta_{k}^{\top} P \\ P A_{L} & P \eta_{k} & P\end{array}\right] \succ 0, \quad \forall \omega_{k} \in \mathbf{B}^{n_{x}+n_{y}}$.

From the equalities $A_{L}=A-L C, \eta_{k}=(E-L F) \omega_{k}$ and $Y=P L$, the equivalent expression follows: 
$\left[\begin{array}{ccc}\beta P & 0 & A^{\top} P-C^{\top} Y^{\top} \\ 0 & \sigma & \omega_{k}^{\top}\left(E^{\top} P-F^{\top} Y^{\top}\right) \\ P A-Y C & (P E-Y F) \omega_{k} & P\end{array}\right] \succ 0$,
$\forall \omega_{k} \in \mathbf{B}^{n_{x}+n_{y}}$.

Since $\omega_{k}$ appears in an affine way in the previous LMI, the inequality is satisfied if and only if it is verified for all the vertices of $\mathbf{B}^{n_{x}+n_{y}}$. This completes the proof.

Remark 1. The center of the ellipsoid $\bar{x}_{k+1}$ (which is the nominal state estimation at time $k+1$ ) is computed like a Luenberger observer which is motivated by the fact that the system is linear. The gain $L=P^{-1} Y$ is obtained after solving LMI (4). Note that when $\beta$ is a free variable in the interval $(0,1)$, expression $(4)$ becomes a very simple case of Bilinear Matrix Inequality (BMI). As $\beta \in(0,1)$ is a bounded scalar, this expression can be rewritten as a LMI problem by successively fixing the value of $\beta$ via the bisection algorithm or using any available BMI solver from the literature (e.g. penbmi solver Kočvara and Stingl (2003)).

Remark 2. A sufficient condition is to choose $\rho_{k+1}=$ $\beta \rho_{k}+\sigma$ in the equation (7), which means to consider the worst case.

Remark 3. At infinity, the sequence $\rho_{k}$ converges to $\rho_{\infty}=$ $\frac{\sigma}{1-\beta}$. Reducing the size of the associated ellipsoid can be done by minimizing $\sigma$ subject to LMI (4) and the additional constraint $\operatorname{tr} P \leq 1$. Another possibility is to impose $P \preceq \mathrm{I}$ as additional constraint.

The proposed optimization problem is solved off-line. Online, it is possible to take advantage of the knowledge of matrix $P$ in order to obtain a different gain $L_{k}$ at each time instant $k$. The online method improves the rapidity of the convergence of the estimation. Given $\bar{x}_{k}$ and $\rho_{k}$, it consists in minimizing the radius $\rho_{k+1}$ at each time instant. This method will be developed in the next section.

\section{ONLINE ELLIPSOIDAL STATE ESTIMATION}

This section proposes a new online method which improves the convergence of the state estimation. In fact, once the matrix $P$ is computed off-line, it is possible to improve the convergence by computing an optimal value for $L_{k}$ at each iteration. The existence of $P, L$, and $\beta$ (initially computed via the off-line method) guarantees the existence of $L_{k}$ satisfying the considered constraints. This method allows minimizing the radius $\rho_{k+1}$ at each iteration. Therefore, it ensures the non-increasing condition of the ellipsoidal radius. The following proposition formulates the proposed online approach.

Theorem 2. Consider that at time instant $k$ the system state $x_{k}$ belongs to the ellipsoid $\mathcal{E}\left(P, \bar{x}_{k}, \rho_{k}\right)$, with the matrix $P$, the radius $\rho_{k}$ and the scalar $\sigma$ computed off-line (using the result of Theorem 1). If there exist a matrix $Y_{k} \in \mathbb{R}^{n_{x} \times n_{y}}$, a scalar $\beta \in(0,1)$ and a radius $\rho_{k+1}$ satisfying the following LMI optimization problem for all $\omega_{k} \in \mathcal{V}_{\mathbf{B}^{n_{x}+n_{y}}}$ :

$\min _{\beta, Y_{k}, \rho_{k+1}} \rho_{k+1}$

subject to

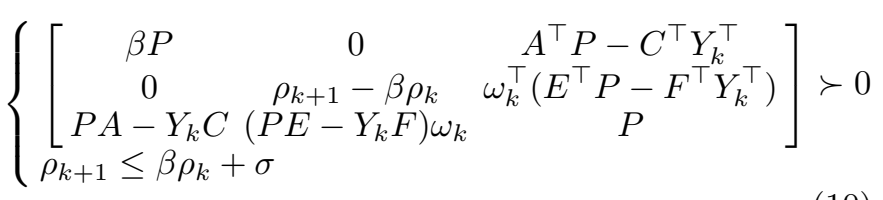

then the system state $x_{k+1}$ at time $k+1$ is guaranteed to belong to the ellipsoid $\mathcal{E}\left(P, \bar{x}_{k+1}, \rho_{k+1}\right)$, for all $\omega_{k} \in$ $\mathbf{B}^{n_{x}+n_{y}}$, with:

$$
\begin{aligned}
P L_{k} & =Y_{k} \\
\bar{x}_{k+1} & =A \bar{x}_{k}+B u_{k}+L_{k}\left(y_{k}-C \bar{x}_{k}-D u_{k}\right) .
\end{aligned}
$$

Proof: The proof is similar to the proof of Theorem 1, replacing $\sigma$ by $\rho_{k+1}-\beta \rho_{k}$ and $L$ by $L_{k}$.

The second constraint $\rho_{k+1} \leq \beta \rho_{k}+\sigma$ of LMIs (10) ensures the non-increasing condition of the ellipsoidal radius. In fact, this implies a reduction on the ellipsoidal radius only if the radius $\rho_{k}$ is larger than $\rho_{\infty}$.

Online verifying the LMI problem (10) for all the vertices of $\mathbf{B}^{n_{x}+n_{y}}$ requires a large computation time and it is suitable to find a technique reducing the number of the vertices to be checked. This will be detailed in the next section.

\section{NEW SCALING TECHNIQUE FOR THE ONLINE ELLIPSOIDAL STATE ESTIMATION METHOD}

To avoid the vertex enumeration and reduce the computation time when solving problem (10) online, a new scaling technique is proposed in this section. This technique is based on the results developed by Alamo et al. (2008) and it will be applied to the LMI optimization problem (10).

The perturbation vector $\omega_{k}$ can be written as: $\omega_{k}=\left[\begin{array}{llll}\omega_{k_{1}} & \omega_{k_{2}} & \cdots & \omega_{k_{n_{x}+n_{y}}}\end{array}\right]^{\top} \in \mathbf{B}^{n_{x}+n_{y}}$. In the elementwise formulation, the following expressions are true:

$\left|\omega_{k}\right| \leq 1$ and $\omega_{k}^{2} \leq 1$.

Denote by $e_{i}$ the columns of the following identity matrix $I_{n_{x}+n_{y}}=\left[\begin{array}{llll}e_{1} & e_{2} & \ldots & e_{n_{x}+n_{y}}\end{array}\right]$. This permits writing: $\omega_{k}^{\top} e_{i} e_{i}^{\top} \omega_{k} \leq 1, \quad i=1, \ldots, n_{x}+n_{y}$. Then, denoting by $T_{i}=e_{i} e_{i}^{\top}$ the matrix having only the element $(i, i)$ equal to 1 , it leads to the following scalar inequalities:

$$
\omega_{k}^{\top} T_{i} \omega_{k} \leq 1, \quad i=1, \ldots, n_{x}+n_{y}
$$

Property 1. Consider a positive definite matrix $S \in$ $\mathbb{R}^{\left(n_{x}+n_{y}\right) \times\left(n_{x}+n_{y}\right)}$ and the positive real scalars $\rho>0$ and $\tau_{i} \geq 0, \quad i=1, \ldots, n_{x}+n_{y}$. If:

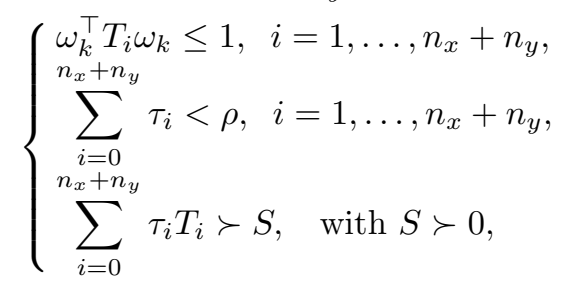

then the following inequality holds $\frac{\omega_{k} \omega_{k}^{\top}}{\rho} \prec S^{-1}$.

Proof: From (14.b), it is trivial to see that: $\rho>\sum_{i=0}^{n_{x}+n_{y}} \tau_{i} \geq 0$. 
Multiplying left and right expression (14.c) by $\omega_{k}^{\top}$ and $\omega_{k}$, respectively, and then using (14.a), leads to:

$$
\begin{gathered}
\omega_{k}^{\top} S \omega_{k}<\omega_{k}^{\top}\left(\sum_{i=0}^{n_{x}+n_{y}} \tau_{i} T_{i}\right) \omega_{k}=\sum_{i=0}^{n_{x}+n_{y}} \tau_{i}\left(\omega_{k}^{\top} T_{i} \omega_{k}\right) \\
\leq \sum_{i=0}^{n_{x}+n_{y}} \tau_{i}<\rho .
\end{gathered}
$$

This can be rewritten as $\rho-\omega_{k}^{\top} S \omega_{k}>0, S \succ 0$ and further reformulated using the Schur complement:

or equivalently:

$$
\left[\begin{array}{cc}
\rho & \omega_{k}^{\top} \\
\omega_{k} & S^{-1}
\end{array}\right] \succ 0, \quad S \succ 0
$$

$$
\left[\begin{array}{cc}
S^{-1} & \omega_{k} \\
\omega_{k}^{\top} & \rho
\end{array}\right] \succ 0, \quad \rho>0 .
$$

Applying again the Schur complement, gives:

$$
S^{-1}-\omega_{k} \rho^{-1} \omega_{k}^{\top} \succ 0, \quad \rho>0 .
$$

which leads to $\frac{\omega_{k} \omega_{k}^{\top}}{\rho} \prec S^{-1}$. This completes the proof.

Applying Property 1 to problem (10), the following property is obtained.

Property 2. If the first LMI constraint of the optimization problem (10) is verified:

$$
\left[\begin{array}{ccc}
\beta P & 0 & A^{\top} P-C^{\top} Y_{k}^{\top} \\
0 & \rho_{k+1}-\beta \rho_{k} & \omega_{k}^{\top}\left(E^{\top} P-F^{\top} Y_{k}^{\top}\right) \\
P A-Y_{k} C & \left(P E-Y_{k} F\right) \omega_{k} & P
\end{array}\right] \succ 0
$$

$\forall \omega_{k} \in \mathcal{V}_{\mathbf{B}^{n_{x}+n_{y}}}$, then there exist a scalar $\beta>0$ and a matrix $S=S^{\top} \in \mathbb{R}^{\left(n_{x}+n_{y}\right) \times\left(n_{x}+n_{y}\right)}$ verifying the constraints (14.a), (14.b) and (14.c) of Property 1 such that:

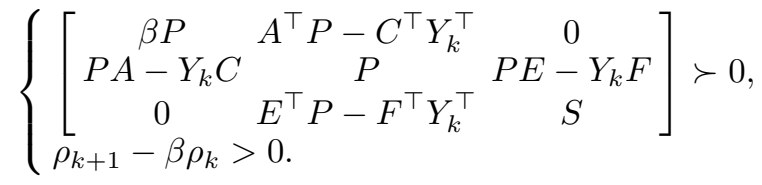

Proof: Pre multiplying and post multiplying inequality (15) by $\left[\begin{array}{lll}I & 0 & 0 \\ 0 & 0 & I \\ 0 & I & 0\end{array}\right]$ leads to:

$$
\left[\begin{array}{ccc}
\beta P & A^{\top} P-C^{\top} Y_{k}^{\top} & 0 \\
P A-Y_{k} C & P & \left(P E-Y_{k} F\right) \omega_{k} \\
0 & \omega_{k}^{\top}\left(E^{\top} P-F^{\top} Y_{k}^{\top}\right) & \rho_{k+1}-\beta \rho_{k}
\end{array}\right] \succ 0
$$

with $\rho_{k+1}-\beta \rho_{k}>0$. Applying the Schur complement and using the notation $P E-Y_{k} F=H_{k}$, gives:

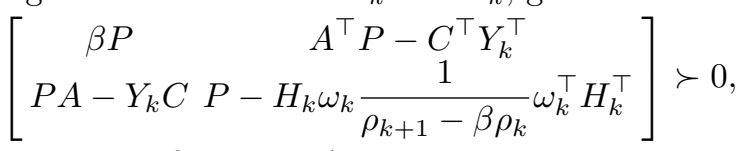

with $\rho_{k+1}-\beta \rho_{k}>0$. Applying Property 1 to the term $\omega_{k} \frac{1}{\rho_{k+1}-\beta \rho_{k}} \omega_{k}^{\top}$, with $\rho=\rho_{k+1}-\beta \rho_{k}$, means that $\exists S=$ $S^{\top} \succ 0$ such that:

or equivalently:

$$
\omega_{k} \frac{1}{\rho_{k+1}-\beta \rho_{k}} \omega_{k}^{\top} \prec S^{-1}
$$

$$
-\omega_{k} \frac{1}{\rho_{k+1}-\beta \rho_{k}} \omega_{k}^{\top} \succ-S^{-1}, \quad \rho_{k+1}-\beta \rho_{k}>0 .
$$

Therefore, if the following expression is verified:

$$
\left[\begin{array}{cc}
\beta P & A^{\top} P-C^{\top} Y_{k}^{\top} \\
P A-Y_{k} C P-\left(P E-Y_{k} F\right) S^{-1}\left(E^{\top} P-F^{\top} Y_{k}^{\top}\right)
\end{array}\right] \succ 0,
$$

with $S \succ 0$, then expression (16) is verified. The constraint (20) can be decomposed as the following:

$$
\begin{gathered}
{\left[\begin{array}{cc}
\beta P & A^{\top} P-C^{\top} Y_{k}^{\top} \\
P A-Y_{k} C & P
\end{array}\right]-\left[\begin{array}{c}
0 \\
P E-Y_{k} F
\end{array}\right] S^{-1}} \\
{\left[\begin{array}{ll}
0 & E^{\top} P-F^{\top} Y_{k}^{\top}
\end{array}\right] \succ 0, \quad S \succ 0 .}
\end{gathered}
$$

Applying the Schur complement leads to expression (16), which completes the proof.

Remark 4. Property 2 permits avoiding the vertex enumeration used in Theorem 2 corresponding to the online ellipsoidal state estimation.

\section{ILLUSTRATIVE EXAMPLE}

Consider the following linear discrete-time invariant system:

$$
\left\{\begin{array}{l}
x_{k+1}=\left[\begin{array}{ccc}
1 & 0 & 0.2 \\
0 & 0.8 & 1 \\
0 & 0 & 1
\end{array}\right] x_{k}+\left[\begin{array}{ccccc}
0.1 & 0 & 0 & 0 & 0 \\
0 & 0.05 & 0 & 0 & 0 \\
0 & 0 & 0.05 & 0 & 0
\end{array}\right] \omega_{k} \\
y_{k}=\left[\begin{array}{ccc}
1 & 0 & 2 \\
0 & -1 & 1
\end{array}\right] x_{k}+\left[\begin{array}{ccccc}
0 & 0 & 0 & 0.15 & 0 \\
0 & 0 & 0 & 0 & 0.15
\end{array}\right] \omega_{k}
\end{array}\right.
$$

with $\left\|\omega_{k}\right\|_{\infty} \leq 1$. The value of $\omega_{k}$ is generated by a random function. The initial state belongs to the ellipsoid $\mathcal{E}\left(I_{3},(0,0,0), \sqrt{2}\right)$ as an arbitrary initialization. In this example, the results obtained by the off-line ellipsoidal state estimation method (detailed in Section 2), the online ellipsoidal state estimation method (detailed in Section 3) and the online method with the scaling technique (detailed in Section 4) are analyzed.

Figures 1, 2 and 3 compare the bounds of $x_{k}$ obtained via the proposed methods: the off-line ellipsoidal estimation method (blue dashed lines), the online ellipsoidal estimation method (magenta dashdot lines), and online ellipsoidal estimation method with the scaling technique (black solid lines). The red stars represent the real state of the system. These points are found between the upper bound and the lower bound of $x_{k}$, which confirms that the bounds are well estimated. The online method offers a faster convergence rate of the estimation than the off-line method (see Fig. 1, 2, 3).

The simulation results have been obtained with an Intel Core $i 7-37703.40 \mathrm{GHz}$, using the LMI solver mincx of MATLAB $^{\mathrm{TM}}$ Robust Control Toolbox.

Figures 4, 5, and 6 illustrate the comparison of the width of the bounds of $x_{k}$ computed by the proposed methods and the off-line $P$-radius-based zonotopic estimation, considering the off-line method as reference. The best accuracy of the estimation is obtained using the online ellipsoidal estimation method. Using the scaling technique will significantly increase the accuracy compared to the off-line $P$-radius-based zonotopic estimation method and reduce the complexity comparing to the online method (see Table 1 ), but with a small loss on the accuracy compared to the results obtained via the online method (Fig. 1, 2, 3). The increase of the bounds width of $x_{k}$ for the $P$-radius-based 


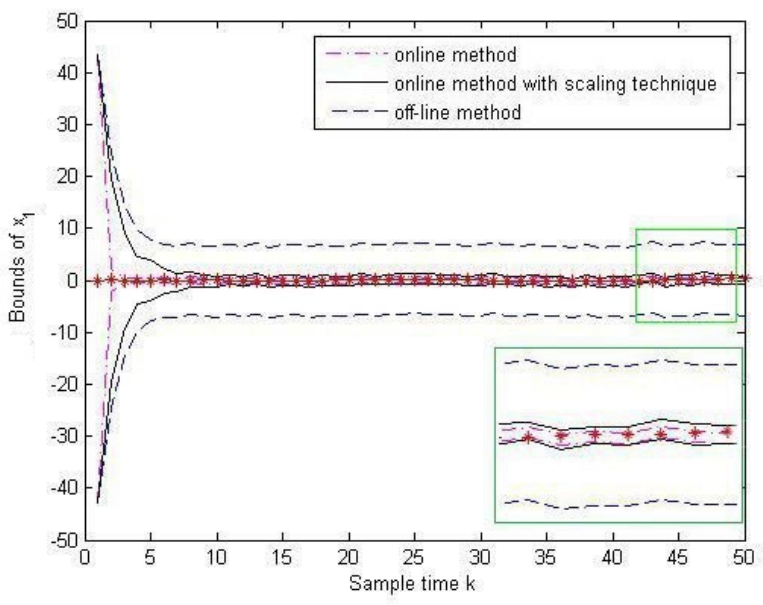

Fig. 1. Bounds on $x_{1}$

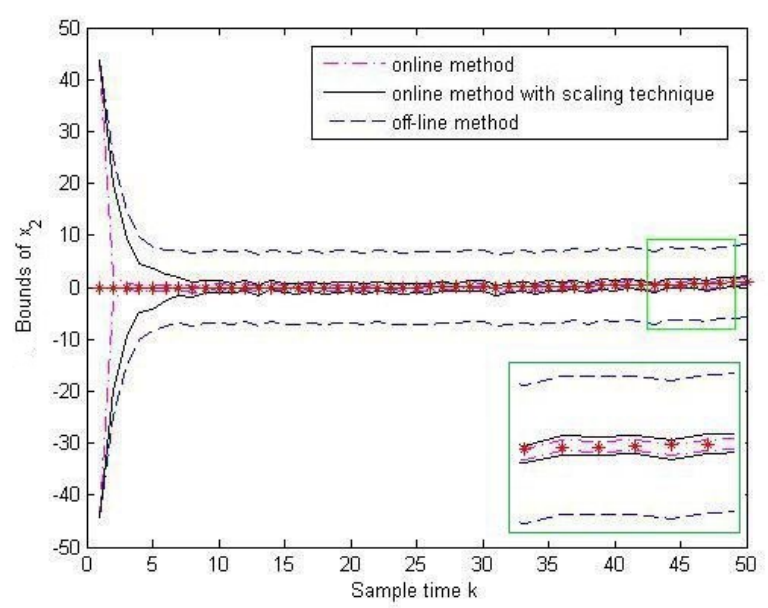

Fig. 2. Bounds on $x_{2}$

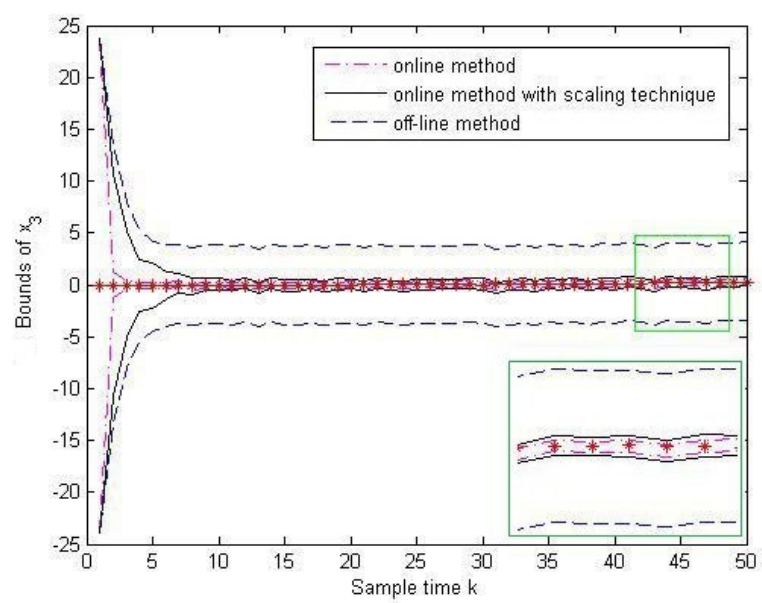

Fig. 3. Bounds on $x_{3}$

zonotopic estimation method at the beginning is related to the rate of convergence of this method. In conclusion, the online estimation method with the scaling technique offers a good trade-off between the accuracy of the ellipsoidal state estimation and the computation time.
Table 1. Total computation time after 50 time instants

\begin{tabular}{lc} 
Algorithm & Time(second) \\
\hline Off-line method (without off-line optimization) & 0.5148 \\
Off-line method (with off-line optimization) & 1.1232 \\
Online method & 7.8625 \\
Online method with scaling technique & 1.7628
\end{tabular}

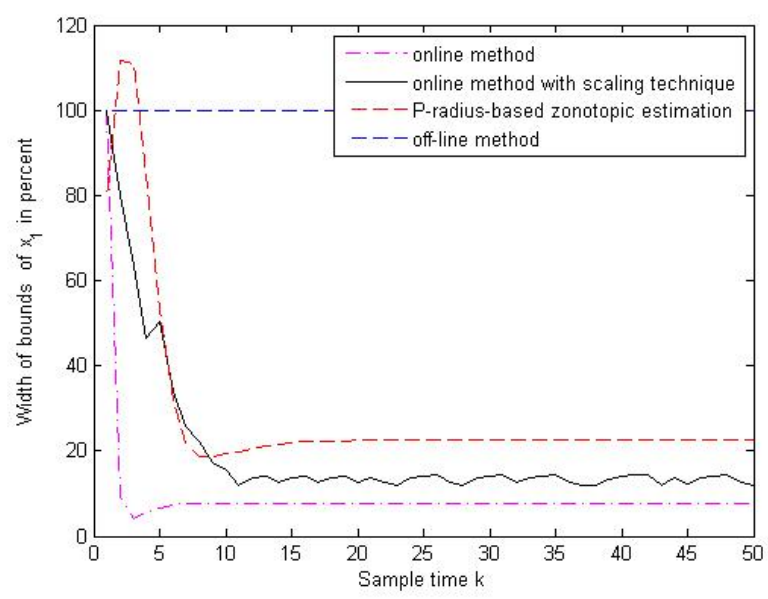

Fig. 4. Comparison of the bound's width of $x_{1}$

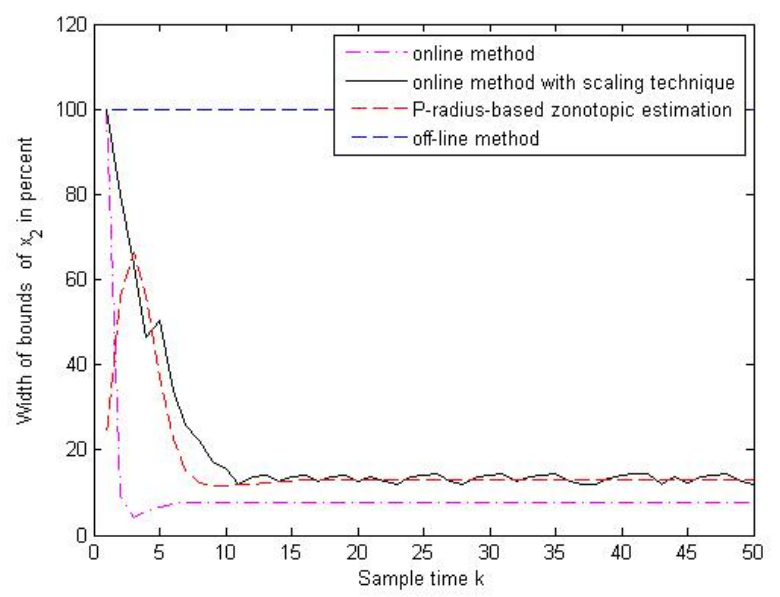

Fig. 5. Comparison of the bound's width of $x_{2}$

Table 2 shows the volume of the ellipsoidal estimation sets at the steady-state obtained by different methods. This confirms that the online method with the scaling technique offers good accuracy compared to the off-line estimation, with a gain on the computation time compared to the online estimation method (see Table 1 ).

Table 2. Comparison of the volume of the estimation sets at the steady-state

\begin{tabular}{lc} 
Algorithm & Volume \\
\hline Off-line method & 8.9666 \\
Online method & 0.0041 \\
Online method with scaling technique & 0.0247
\end{tabular}

\section{CONCLUSION}

A new approach to guaranteed ellipsoidal state estimation for multivariable linear discrete-time systems with 


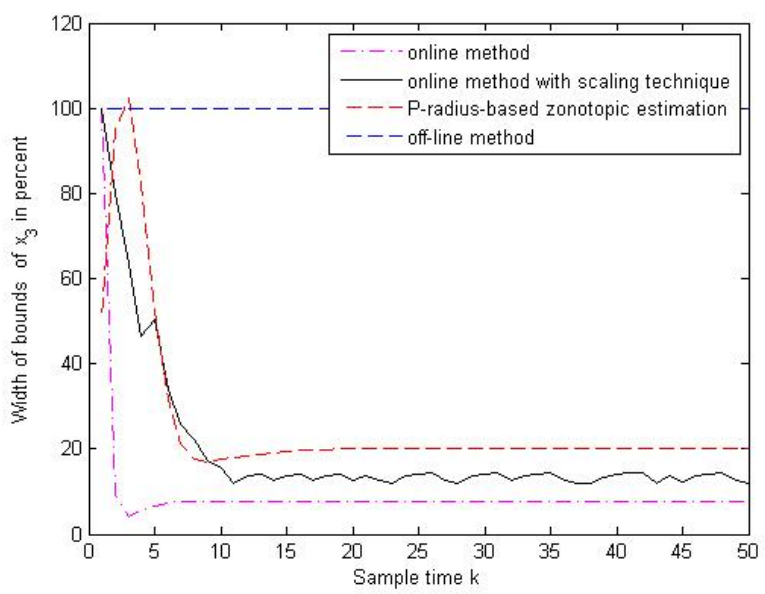

Fig. 6. Comparison of the bound's width of $x_{3}$

bounded perturbations and measurement noise has been proposed. The proposed approach bounds the set of the system states that are consistent with the output measurement by an ellipsoid. First, this ellipsoidal set is minimized at each iteration by off-line solving an LMI problem. A new online method is further proposed to improve the accuracy of the estimation. Using a new scaling technique together with the online method, the computation time is significantly reduced, while keeping an acceptable level of the estimation accuracy. An example illustrates the performance of the proposed methods.

Two interesting perspectives are to extend these methods to the case of systems with interval uncertainties and to apply them to fault detection and fault tolerant control purposes.

\section{APPENDIX}

$S$-procedure for quadratic functions and nonstrict inequalities (see Boyd et al. (1994)): Let $F_{0}, \ldots, F_{p}$ be quadratic functions of variable $\zeta \in \mathbb{R}^{n}: F_{i}(\zeta) \triangleq \zeta^{\top} T_{i} \zeta+2 \mu_{i}^{\top} \zeta+v_{i}$, with $i=0, \ldots, p$ and $T_{i}=T_{i}^{\top}$. Consider:

$$
F_{0}(\zeta) \geq 0 \text { for all } \zeta \text { such that } F_{i}(\zeta) \geq 0, i=1, \ldots, p
$$

If $\exists \tau_{i} \geq 0, i=1, \ldots, p$, such that $F_{0}(\zeta)-\sum_{i=1}^{p} \tau_{i} F_{i}(\zeta) \geq 0$ for all $\zeta$, then expression $(22)$ holds. It is a non trivial fact that when $p=1$, the converse holds meaning that there is some $\zeta_{0}$ such that $F_{0}\left(\zeta_{0}\right) \geq 0$.

\section{REFERENCES}

Alamo, T., Bravo, J.M., and Camacho, E.F. (2005). Guaranteed state estimation by zonotopes. Automatica, 41, $1035-1043$

Alamo, T., Tempo, R., Ramirez, D.R., and Camacho, E.F. (2008). A new vertex result for robustness problems with interval matrix uncertainty. Systems and Control Letters, 57, 474-481.

Bertsekas, D.P. and Rhodes, I.B. (1971). Recursive state estimation for a set-membership description of uncertainty. IEEE Transaction on Automatic Control, 16(2), $117-128$
Boyd, S., ElGhaoui, L., Feron, E., and Balakrishnan, V. (1994). Linear Matrix Inequalities in System and Control Theory. SIAM, Philadelphia.

Chernousko, F.L. (1994). State Estimation for Dynamic Systems. CRC Press, Boca Raton.

Chisci, L., Garulli, A., and Zappa, G. (1996). Recursive state bounding by parallelotopes. Automatica, 32, 10491055 .

Combastel, C. (2003). A state bounding observer based on zonotopes. In Proc. of European Control Conference. Cambridge, UK.

Daryin, A.N. and Kurzhanski, A.B. (2012). Estimation of reachability sets for large-scale uncertain systems: from theory to computation. In Proc. of 51st IEEE Conference on Decision and Control, Maui, Hawaii, USA, 7401-7406.

Daryin, A.N., Kurzhanski, A.B., and Vostrikov, I.V. (2006). Reachability approaches and ellipsoidal techniques for closed-loop control of oscillating systems under uncertainty. In Proc. of 51st IEEE Conference on Decision and Control, San Diego, CA, USA, 6390-6395.

Durieu, C., Walter, E., and Polyak, B. (2001). MultiInput Multi-Output ellipsoidal state bounding. Journal of Optimization Theory and Applications, 111(2), 273303.

Fogel, E. and Huang, Y.F. (1982). On the value of information in system identification-bounded noise case. Automatica, 18, 229-238.

$\mathrm{Hu}, \mathrm{T}$. and Lin, Z. (2003). Composite quadratic Lyapunov functions for constrained control systems. IEEE Transaction on Automatic Control, 48, 440-450.

Kalman, R.E. (1960). A new approach to linear filtering and prediction problems. Transactions of the ASMEJournal of Basic Engineering, 82(Series D), 35-45.

Kočvara, M. and Stingl, S. (2003). PENNON A Code for convex nonlinear and semidefinite programming. optimization methods and software, 18(3), 317-333.

Kurzhanski, A.B. and Vályi, I. (1996). Ellipsoidal Calculus for Estimation and Control. Birkhaüser Boston.

Le, V.T.H., Stoica, C., Alamo, T., Camacho, E.F., and Dumur, D. (2013). Zonotopic guaranteed state estimation for uncertain systems. Automatica, 49(1), 3418-3424.

Polyak, B., Nazin, S.A., Durieu, C., and Walter, E. (2004). Ellipsoidal parameter or state estimation under model uncertainty. Automatica, 40, 1171-1179.

Puig, V., Cugueró, P., and Quevedo, J. (2001). Worst-case state estimation and simulation of uncertain discretetime systems using zonotopes. In Proc. of European Control Conference. Portugal.

Schweppe, F.C. (1968). Recursive state estimation: Unknown but bounded errors and system inputs. IEEE Transaction on Automatic Control, 13(1), 22-28.

Walter, E. and Piet-Lahanier, H. (1989). Exact recursive polyhedral description of the feasible parameter set for bounded-error models. IEEE Transaction on Automatic Control, 34(8), 911-915.

Wistenhausen, H.S. (1968). Sets of possible states of linear systems given perturbed observations. IEEE Transaction on Automatic Control, 13(5), 556-558. 